\title{
The health and performance benefits of the squat, deadlift, and bench press
}

\begin{abstract}
This article explores the squat, deadlift and bench press prescribed by fitness trainers, strength and conditioning coaches and rehabilitation therapists to develop whole body strength, improve health outcomes or to rehabilitate the upper or lower limbs following injury. More specifically, the purpose of this article is to review the health and performance benefits of the squat, deadlift and bench press.
\end{abstract}

Keywords: squat mechanics, rehabilitation, gluteus, bulgarian, knee flexion

\author{
Volume 3 Issue 2 - 2018 \\ Luke Del Vecchio,' Hays Daewoud,' Shannon \\ Green ${ }^{2}$ \\ 'Department of Australian Combat \& Exercise, Australia \\ ${ }^{2}$ Department of Warrior Performance, Australia
}

Correspondence: Luke Del Vecchio, Department of Australian Combat \& Exercise,Australia Email I.delvecchio@cqu.edu.au

Received: March 20, 2018 | Published: April 06, 2018

\section{Introduction}

\section{The squat}

The squat is a well-known exercise to strengthen the muscles of the lower limb. Conditioning specialists universally agree the squat is among the top three prescribed exercises for sports training, rehabilitation and prehabilitation. ${ }^{1}$ Previously, there has been much debate about the safety of deep (full range) squats. Strength and conditioning coaches often define a deep squat by amount knee range of motion (120-140 degrees), or by observation of thigh position, which during a deep squat is below parallel to the floor. Historically, some researchers ${ }^{2}$ have raised concerns about the levels of knee compressive forces that occur during full range squats, however this concern has been rejected by leading strength and conditioning authorities such as the National Strength and Conditioning Association. In the NSCA Position Paper on the squat exercise, the NCSA has rejected these concerns, providing substantial scientific evidence that full range of motion squats do not expose the knee to damaging compressive forces. ${ }^{3}$ Interestingly, researchers have reported knee joint compression may be higher in the partial range of motion squat, as peak patellofemoral joints experience peak forces at 90-100 degrees of knee flexion during the squat. ${ }^{4}$ According to Chandler and Stone the squat when performed correctly regardless of depth, and with appropriate supervision, not only is safe but maybe a significant deterrent to knee injuries. Importantly, however, ${ }^{4}$ also report that bouncing out of the bottom position of a squat, to help initiate the ascent increases the mechanical load on the knee joint. This is because the highest forces and muscular tension during a squat typically occur at the transition from descent (eccentric) to ascent (concentric), as muscle lengthening is much higher during full range of motion squats. Altogether, these data suggest deep squats, when adequately supervised, do not impose damaging forces to the knees.

\section{Squat depth}

The discussion on deep or partial range squatting also extends into athletic performance. Some researchers believe ${ }^{5}$ deep squats may be more beneficial for vertical jump performance, than partial range squats. In contrast, other researchers have shown that maximal strength in partial range of motion squats is a strong determinant of sprint and vertical jump performance. ${ }^{6}$ When compared to a deep squat $^{7}$ reported a partial range of motion squat, provide greater transfer to improvements in both sprinting and jumping ability, including partial range of motion squats in a conditioning session can result in more significant improvement in sporting skills. However, the authors of this study acknowledge that deep squats maybe useful for general sports conditioning programs. The likely explanation for the observed improvement in sprint and jump performance from partial range of motion squats likely due to similar hip and knee joint ranges observed in both sprinting and jumping. However, partial range of motion squats is not without limitations. For example, in their recent case study, reported a preference for deep squats when training their female volleyball players. The authors of this study stated the volleyball players when prescribed partial range of motion squats complained that the more substantial loads (1-1.2x body weight) increased the discomfort on their backs and knees. Taken together, the results of these studies suggest there are benefits to both full and partial range of motion squats. Full range of motion squats are likely to provide general conditioning benefits, and may also be beneficial for improving vertical jump performance. Whereas partial range of motion squats appear to provide specific benefits to both sprinting and vertical jumping performance, however, due to the higher loads that can be utilized with quarter range of motion squats, there may be limitations such as additional hip and knee joint stress.

\section{Squat mechanics}

Biomechanically, the squat is a closed-chain movement, requiring simultaneous extension patterns of the ankle, knee and hip joints. The squat can be performed in many different ways, including variations in foot width (sumo squats), foot position (single leg squats, Bulgarian squats), load position (front squats, sissy squats) and depth (full range squats, shallow squats). However, it is essential to keep in mind that each one of these variations will influence knee joint forces and muscle recruitment patterns. The squat is a highly versatile exercise that can target specific muscles groups for both performance and rehabilitation purposes. Knowing how squat variants, affect muscle recruitment patterns, can assist fitness instructors and coaches to modify the squat, to target specific muscles during both rehabilitation and strengthening programs. 


\section{Muscle mechanics}

\section{Glute activity}

Research which has investigated the effects of squat depth on gluteus maximus activity have found that gluteus maximus activity increases with depth. ${ }^{8}$ However, these results appear to vary with different loads (\% of 1RM). Other researchers have found Gluteus maximus recruitment may increase with increases in squat stance width. For example, observed a significant increase in gluteus maximus activity when squats were performed with wider stances. Finally, ${ }^{9}$ analyzed the back squat and the overhead squat and found; the back squat elicited greater gluteus maximus activity than the overhead squat. Interestingly, when compared to the front, full, or parallel squats, squatting at full range, did not elicit greater Gluteus maximus activation, suggesting either front, full, or parallel squats are equally effective exercises for Glut development (Contreras, Vigotsky, Schoenfeld. In conclusion, the results from these studies suggest the squat is an excellent exercise to strengthen the musculature of lower limbs. Also, muscle activity during a squat can be affected foot position, depth, support, and load.

\section{Quadriceps activity}

During a squat, the quadriceps are the prime movers, particularly the vasti muscle group, which show significantly higher activity than the rectus femoris. Peak quadriceps activity occurs at 80-90 degrees of a squat, with no further increases with greater knee flexion. ${ }^{10}$ This data indicates, half squats (to 90 degrees of knee flexion) will maximize quadriceps activity. Descending beyond 90 degrees of knee flexion, which is near the parallel squat position, may not enhance quadriceps development. ${ }^{10}$ Finally, when compared to hack squatmachines, wall squats with hip support, appear to increase quadriceps activity. ${ }^{11}$ Also, this researcher observed greater quadriceps activity when performing squats on both the hack squat-machine and against a wall. Taken together, these data suggest when, squatting to smaller ranges of motion, placing the feet forward or performing squats against a wall with hip support may be a more efficient means of targeting quadriceps.

\section{Vastus medial obliquus (VMO)}

The vastus medial obliquus muscle is the most distal segment of the vastus medial muscle. Its specific training plays a major role in maintaining patella position and limiting injuries to the knee. ${ }^{12}$ Weakness, timing, and dysfunction of the VMO cause mal-tracking of the patella and subsequent damage to surrounding structures which leads to increased forces on the knees, often resulting in injuries. ${ }^{13}$ Furthermore, imbalances between vastus lateral and VMO enhance the risk for patellofemoral pain, ${ }^{14}$ this data demonstrates the importance of early VMO training following a knee injury. Research on $\mathrm{VMO}$ activity during a squat shows the VMO contributes $30.88 \%$ to the activity of the thigh during the partial squat; yet, it contributes only 18.85 and $20.23 \%$ during the parallel and full squats. ${ }^{15}$ Other research ${ }^{16}$ has investigated if wider foot positions affect VMO activity relative to VL activity (VMO: VL ratio). The researchers reported a wider foot position does not increase VMO activity. However VMO was more active throughout a $90^{\circ}$ range, and increasing knee flexion angles can increase the activity of the VMO relative to the VL. Taken together, these findings suggest squatting to no greater than 90 degrees of knee flexion; may be the optimal squat depth for VMO.

\section{Hamstrings}

The hamstrings due to their biarticular nature act eccentrically during the descent, and concentrically during the ascent. However as the knee flexes during the descent, the hip flexes, the length of the hamstrings is maintained throughout the squat; resulting in a minimal change in hamstring length. The lack of change in hamstring length during a squat can increase the length-tension relationship in favor of force production. ${ }^{17}$ Research suggests hamstring activity is highest during the ascent phase of a squat and is strongly related to weight lifted. ${ }^{18}$ Hamstring activity during a squat; reaches peak activity between 50-70 degrees of knee flexion. In contrast, during a bodyweight squat, hamstring activity is minimal, and not significant until loads reach approximately 12 repetition maximum (RM), presumably to enhance knee stability. Finally, other research has ${ }^{19}$ observed a significant increase in hamstring activity when squats are performed (a) in a squat hack machine and (b) as a wall squat, with scapular support, and with the feet position forward of center mass. Interestingly, both deep squats and half squats appear to stimulate hamstring activity equally. Taken together, these results indicate that these aforementioned variations of the squat, efficiently stimulate hamstring activity.

\section{Calf muscle activity}

Previous research which has investigated calf muscle activity and force during a squat has observed a moderate amount of calf muscle activity. Calf muscle activity (Gastrocnemius) increases as the knee progressively flexes during the decent phase; and decreases during the ascent, as knee extension increases. Calf (Gastrocnemius) activity appears to peak between 60 to 90 degrees of knee flexion; to eccentrically control the rate of ankle dorsiflexion during the descent. Finally, positioning the feet directly under the hips during a wall slide squat has been shown to increase calf muscle activity. Altogether, these data suggest calf muscle activity can be increased by performing squats with the feet forward of center mass and restricting knee flexion range of motion to 60-90 degrees.

\section{The squat - benefits, and application to sport and activities of daily living}

The squat is an ideal exercise to strengthen the entire lower limb for both sporting and ADLs. For example, increased squatting strength is strongly linked to sports performance..$^{20}$ For example, increased strength in a partial range of motion squat may have a positive transfer to sprinting and vertical jumping performance. ${ }^{21}$ Whereas, increased strength in the deep squat, may have a positive transfer vertical jump performance. ${ }^{22}$ The squat due to its ability to mimic many different activities of daily living is an ideal choice for general health and fitness training programs. For example, previous research has shown using simple bodyweight squats as part of a strength training intervention has been shown to improve the performance of activities of daily living in older individuals. ${ }^{23}$ Also, the squat as part of a good balance resistance training program can build lower limb strength and lean mass, helping counteract age-related pathologies such as sarcopenia and dynapenia. ${ }^{24}$ However, the use of the squat for specific situations may be unique to the individual and task at hand. Outlined below are some examples of both sporting and ADLs tasks that may benefit.

\section{The deadlift}

The deadlift is a simple and functional exercise that requires force application and transfer through the entire kinetic chain. ${ }^{25}$ The 
Deadlift involves the simultaneous activation of multiple muscle groups throughout the entire body; this coordinated effort results in a significant amount of stress on the musculoskeletal system that may initiate several different systematic adaptations. Historically, athletes and non-athletes alike have used deadlift, to strengthen the hip, thigh, and back musculature. ${ }^{26}$ Interestingly, many strength coaches believe the squat and deadlift have very similar characteristics. ${ }^{27}$ To the naked eye, the squat and deadlift appear very similar (both exercises involve triple joint extension). However, from a biomechanical point of view, the two lifts are in fact, entirely different. ${ }^{28}$ For example, compared to the squat, the deadlift requires force production without the aid of the stretch-shortening cycling, so unlike the squat; the deadlift starts with a concentric contraction and ends with an eccentric contraction. ${ }^{29}$ Thus, the squat differs because, it allows viscoelastic energy stored in the muscles and tendons on the way down, to be expressed, on the way up. In contrast, the deadlift, however, the deadlift starts in a mechanically difficult position, requiring the lifter to generate the entire force needed to move the bar off the floor, without the assistance of the stretch-shortening cycle..$^{30}$ Finally, in the deadlift, the load is applied perpendicular to the body and loaded in the horizontally plane, unlike the squat, which is a loaded in the vertical plane. ${ }^{31}$ Horizontally loaded movements may be more beneficial for horizontally based sporting movements such as sprinting and long jump, suggesting deadlifts may provide a more significant transfer effect to movements crucial to sports performance. ${ }^{32}$ Taken as a whole, the deadlift appears to be an essential whole body strengthening exercise, which has serval distinct biomechanical differences to the squat, and potential benefit to sporting performance.

\section{Deadlift mechanics}

The deadlift, possess several unique mechanical characteristics that can improve functional strength and movement efficiency and thus the performance of many different physical tasks. The mechanical characteristics of the deadlift, include the ability to recruit significant muscle groups, at high contraction rates. ${ }^{33}$ Also, the deadlift possesses a favorable kinetic profile that allows for continued acceleration through a significant portion of the lift, which is vital for power development. ${ }^{34}$ Together, these data suggest the deadlift may possess several unique mechanical characteristics, which are favorable for the development of functional strength and sporting performance. Traditionally, Lifting technique for the deadlift has been broken down into to two distinct techniques. ${ }^{35}$ The first lifting strategy, known as the "leg-lift" utilizes a bent leg position (knee flexion) with a mostly vertical trunk position. This technique utilizes similar joint angle changes at the hip, knee, and ankle and more closely resembles a squat. ${ }^{36}$ In contrast, the "back-lift" technique involves an extended knee and flexed trunk position during the initial lifting phase of the deadlift. During the back-lift technique, the hips rise more rapidly than the leg-lift, creating an increase in the trunk angle. ${ }^{37}$ According to $^{38}$ the leg-lifting technique imposes lower loads on the lumbar spine, which may be more beneficial for reducing the risk of lower back injury. However, this lifting technique imposes a significant load on the knees. Conversely, the back-lift style exposes the lumbar spine to significantly greater loads. Interestingly, depending on the lifting technique, the lower limb muscle groups are recruited synergistically (leg-lift technique) or in a sequential manner (back-lift), to create the necessary muscle forces to lift the bar off the ground ${ }^{39}$ Intriguingly, researchers ${ }^{40}$ observed weight-trained athletes, when performing heavy deadlifts, change their mid-lift technique, from a leg-lift to a back-lift technique, due to an inability to maintain lumbar lordosis.
Furthermore, some other researchers ${ }^{41}$ have reported the leg-lift is not the preferred deadlifting technique for lifting heavier loads. In summary, deadlifting appears to involve a combination of leg dominant and back dominant lifting technique, depending on the lifting load. The conventional deadlift involves movement patterns (hip hinge) which occur in activities of daily living (lifting objects of the floor), involves a significant number of muscle groups in both the upper and lower body. ${ }^{42}$ Also, the deadlift is routinely performed by athletes in both strength-based and non-strength based disciplines alike. During a deadlift, the lifter commences the lift in a squat position with the knees and hips flexed approximately $80-100$ degrees. ${ }^{43}$ The arms are held straight and pointing down, and a standard or alternating hand grip is used to hold a barbell positioned in front of the lifter's feet. The barbell is then lifted upward in a continuous motion by extending the knees and hips until the lifter is standing erect with knees locked and the shoulders thrust back. From this position, the barbell is slowly lowered back he lifter in a squat position with the knees and hips flexed ${ }^{44}$ Considering the complexity of this exercise, it is essential to understand some of the key anatomical features of this exercise. Generally speaking, the deadlift utilizes the muscles of the lower limb, trunk, and upper limb. More specifically, at the commencement of the lift the gastrocnemius and soleus undergo concentric contraction to plantar flex the ankle as the bar continues to be raised, the quadriceps contract concentrically, to extend the knee as the bar is raised. ${ }^{45}$ At the hip, the gluteus maximus and begin to contract as knee extension is completed. In the trunk, the oblique's and rectus abdominals contract to maintain spinal stability, while the erector spine deep spinal extensors undergo concentric contraction to bring the spine into an upright position. Finally, the scapula stabilizers contract isometrically to prevent upper back flexion, while the latissimus dorsi plays a significant role in stabilizing the bar, preventing it from rolling forward during lift-off. ${ }^{46}$ Interestingly, little research has investigated the muscle recruitment levels of the upper limbs, including the forearm muscles. Anactodately, strength and conditioning coaches believe grip strength plays a vital role in overall deadlift performance, however, to the best of our knowledge, no research to date has reported on the relationship between grip strength and deadlift performance. Together, these results suggest the deadlift recruits many large muscle groups in the lower body and trunk, making it an ideal exercise selection when targeting whole body strength.

\section{Deadlift variations-the sumo deadlift}

The Sumo Deadlift, a variation of deadlift performed with a wide stance, and hand placement inside the knees (as opposed to outside the knees in a conventional deadlift). The sumo deadlift is considered by some researchers to be a more biomechanically efficient lifting technique, due to the shorter distance the bar travels. Also, the sumodeadlift, due to its more upright back position may reduce shearing forces on the lower back. ${ }^{47}$ Previous research has shown in comparison to a conventional deadlift; the sumo-deadlift activates both the medial and lateral quadriceps (values medial and lateral) and, the anterior tibialis. In contrast, the conventional deadlift showed greater, calve (medial gastrocnemius) and erector spinae activity. ${ }^{48}$ Interestingly, research suggests hamstring and glute muscle recruitment does not differ between techniques. In summary, it appears the significant difference between sumo and the conventional difference lies in the quadriceps and calve muscle recruitment, with the sumo deadlift appearing to more efficiently target the quadriceps and tibialis anterior, while the conventional deadlift is appearing to be more efficient for calving muscle recruitment. Taken together, the results of the studies 
suggest suitable differences in muscle recruitment exist between the conventional and sumo deadlift, the sumo deadlift with its lower spinal loads and shorter bar path potentially offering a more suitable deadlifting technique for those with a history of back pain (Table 1).

Table I Outlined below are some examples of both sporting and ADLs tasks that may benefit

\begin{tabular}{|c|c|c|}
\hline $\begin{array}{l}\text { Physiological response/ } \\
\text { benefit }\end{array}$ & Sporting benefit & ADL benefit \\
\hline $\begin{array}{l}\text { Hypertrophy of the hip, thigh } \\
\text { and calve musculature. }\end{array}$ & $\begin{array}{l}\text { Increases effective mass and } \\
\text { functional hypertrophy for contact } \\
\text { sports. }\end{array}$ & $\begin{array}{l}\text { Prevention of sarcopenia in older adults. Assists with chair } \\
\text { raising tasks, and lowering of heavy items to the floor. }\end{array}$ \\
\hline $\begin{array}{l}\text { May increase or prevent } \\
\text { a decline in bone mineral } \\
\text { density. }\end{array}$ & \multicolumn{2}{|c|}{$\begin{array}{l}\text { Important for Rugby League, Rugby Union and other contact sports to buffer collisions and contact. } \\
\text { Assist speed/power athletes with performance without the additional total body mass. }\end{array}$} \\
\hline Leg and Hip strengthening & $\begin{array}{l}\text { Sprint speed, vertical jump } \\
\text { performance. }\end{array}$ & $\begin{array}{l}\text { Prevention of dynapenia in older adults. Assistance with chair } \\
\text { raising ability, stair climbing function. }\end{array}$ \\
\hline Rehabilitation & $\begin{array}{l}\text { VMO strengthening as part of ACL } \\
\text { rehabilitation. }\end{array}$ & Lower limb strengthening after knee replacement surgery. \\
\hline Prehabilitation & $\begin{array}{l}\text { Decrease the risk of ankle, knee and } \\
\text { leg overuse injuries. }\end{array}$ & $\begin{array}{l}\text { Prevention of muscle atrophy prior to surgery. Reduce falls } \\
\text { risk. }\end{array}$ \\
\hline Core Strength & \multicolumn{2}{|c|}{$\begin{array}{l}\text { Significant activation of the abdominals, oblique's and erector spinae muscles if squat loads are }>80 \% \\
\text { 1RM. }\end{array}$} \\
\hline
\end{tabular}

\section{Muscle mechanics}

\section{Gluteus maximus activity}

Athletes and recreational fitness enthusiasts alike commonly use the deadlift in their training/rehabilitation programs for gluteal development. To the best of our knowledge, very few studies have examined the full extent of gluteal activity during a barbell deadlift found gluteus maximus activity was higher when knee flexion angles approached 61-90 degrees compared to lower knee flexion angles (0-30 degrees) during a barbell deadlift. Similarly, ${ }^{49}$ found gluteus maximus activity during a barbell deadlift peaked at $83 \%$ of the lift height. Together, these data demonstrate the significant involvement of the gluteus maximus activity during a deadlift. Due to its role as a hip extensor, it appears glute activity occurs towards the end stages of the lifting phase.

\section{Quadriceps activity}

The deadlift is not usually selected as a primary exercise for quadriceps development. Research from observed higher Quadriceps activity in the conventional barbell deadlift when compared to stiff-legged deadlifts. Quadriceps activity during a barbell deadlift, however, appears to be lower than more quad dominant exercises such as knee extensions, barbell squats, lunges and step ups. ${ }^{50}$ Has also observed significant quadriceps activity during the deadlift. What these results suggest, is the barbell deadlift can be considered as an accessory or additional exercise to strengthen the quadriceps.

\section{Hamstring activity}

Hamstring activity peaks during the beginning of the lift phase (concentric) of the deadlift, when hip extension angles are between 20 and 40 degrees. This peak in hamstring activity is most likely due to its role as a hip extensor in this movement. ${ }^{51}$ Outside of these three studies, there have been very few studies examining hamstring involvement in the deadlift. According to the findings of hamstring activity during a conventional barbell deadlift is more significant than compound lower body exercises such as barbell squats, dumbbell lunges, and step ups. Somewhat surprisingly, found no difference in hamstring involvement between stiff-legged deadlifts and conventional barbell deadlifts. Altogether, these data highlight the substantial involvement of the hamstrings in a conventional barbell deadlift.

\section{Calf muscle activity}

While the barbell deadlift is not often an immediate exercise choice for calf muscle development, two previous studies ${ }^{52}$ have observed a moderate amount of calf muscle involvement during a conventional barbell deadlift. Escamilla compared the involvement of the calf muscles during a conventional barbell deadlift and a sumo deadlift and found calf muscle activity was higher during the conventional barbell deadlift. Compared the involvement of the calf muscles during a stiff-legged deadlift and conventional barbell deadlift and found calf muscle activity was more significant in the stiff-legged deadlift. Together, the results of these studies demonstrate the involvement of the calf muscles in the conventional barbell deadlift.

\section{Core and upper body muscle activity}

The deadlift, unlike the squat, requires significant involvement of the upper limb musculature along with the trunk muscles. ${ }^{53}$ For instance, observed substantial activity in the core muscles during a conventional barbell deadlift. Also, reported core muscle activation was more significant in deadlifts performed at $80 \%$ of $1 \mathrm{RM}$, than either the side plank or superman exercises. In the upper body, noted significant activity of the upper trapezius muscles and observed significant activity in the latissimus dorsi muscles during the conventional barbell deadlift. Taken together, these data demonstrate the significant involvement of the core and upper body muscles during a barbell deadlift.

\section{The deadlift-benefits and application to sport and activities of daily living}

The deadlift with its capacity to train some significant muscle groups, functionally, may offer some benefits to both sporting and the performance of some different ADLs. For example, barbell deadlifts have been shown to improve vertical jump performance and the rate of muscular force production..$^{54}$ Also, the deadlift generates moderate to 
high co-contraction of the quadriceps, hamstrings, and gastrocnemius, making it an ideal close-kinetic exercise for knee rehabilitation. Also, the deadlift has featured in various rehabilitation programs. For example, demonstrated an increase in the capacity to perform activities of daily living following bariatric surgery following several weeks of resistance training that featured the deadlift. Furthermore, reported a significant increase in bone mineral density in a group of younger men and women, following 24 weeks of resistance training also featuring the deadlift. It should be noted, the use of the deadlift for specific situations it may be unique to the individual and task at hand. Outlined below are some examples of both sporting and ADLs tasks that may benefit.

\section{The bench press}

The bench press has long been part of recreational exercise enthusiasts and competitive athlete's strength and conditioning programs. The bench press has been one the most popular upper body strengthening exercises among athletes, bodybuilders and recreational fitness enthusiasts for a long time. The bench press is most commonly performed on a flat bench, with the lifter lying on their back, and their feet placed flat on the floor. Lifters use a shoulder-width grip, commencing the lift by lowering the bar to the chest and then lifting the bar up until the arms are fully extended. It has been suggested ${ }^{55}$ that among the many variations that affect bench press performance, grip width may be one of the most influential aspects. The effect of bench grip width on bench press performance has been a hotly debated topic for some time. Most research suggests wider grip widths, produce the best bench press performance. For example, in one study, vanden demonstrated superior bench press performance with broader grips ( $>1.5 \mathrm{x}$ bi-acromial width). A wider grip is often chosen by competitive lifters, as it reduces the distance of bar travel. Interestingly, the bar travels almost $25 \%$ further with a standard grip width when compared to a grip double the width. However, it should also be known that wider grips increase the risk of pectoralis tears, shoulder instability, atraumatic osteolytic of the distal clavicle. Reducing grip width to $<1.5 \mathrm{x}$ biacromial width can significantly reduce the risk of such injuries. ${ }^{56}$ Lifting speed and bar path are essential to bench press performance. For example, elite powerlifting athletes have been found to perform the bench press with a much lower lifting tempo and lift the bar more in line with the shoulders than recreational lifters. From a safety perspective, it is essential that bar remain horizontal at all times during the lift. Together, the results of these studies suggest correct grip width placement, lifting speed and bar path are essential contributors to successful bench press performance. Upper body strength can also be developed with many variations of the bench press. Common variations include dumbbell chest press, smith machine press, and machine chest presses. However, research has shown loads used with a dumbbell chest press can be approximately $63-83 \%$ of maximal barbell loads. Fitness instructors and coaches in the field, should be aware of these differences when prescribing training loads for these variants of the bench press (Table 2).

Table 2 Outlined below are some examples of both sporting and ADLs tasks that may benefit

\begin{tabular}{|c|c|c|}
\hline $\begin{array}{l}\text { Physiological } \\
\text { Response/Benefit }\end{array}$ & Sporting Benefit & ADL/General Benefit \\
\hline $\begin{array}{l}\text { Hypertrophy of the } \\
\text { hip, thigh and calve } \\
\text { musculature. }\end{array}$ & $\begin{array}{l}\text { Increases effective mass and functional hypertrophy for contact } \\
\text { sports. }\end{array}$ & Prevention of sarcopenia in older adults. \\
\hline $\begin{array}{l}\text { May increase or prevent } \\
\text { a decline in bone } \\
\text { mineral density. }\end{array}$ & $\begin{array}{l}\text { Important for Rugby League, Rugby Union and other contact } \\
\text { sports buffer collisions and contact. Assist speed/power athletes } \\
\text { with performance without the additional total body mass. }\end{array}$ & $\begin{array}{l}\text { Prevention/treatment for low bone } \\
\text { mineral density. }\end{array}$ \\
\hline $\begin{array}{l}\text { Whole body strength, } \\
\text { power and sports } \\
\text { performance }\end{array}$ & Vertical jump performance, explosive strength, sprint speed. & $\begin{array}{l}\text { Picking up, carrying and lowering } \\
\text { household items, grip strength. }\end{array}$ \\
\hline Rehabilitation & $\mathrm{ACL}$ injury/reconstruction & Mechanical lower back pain \\
\hline Prehabilitation & $\begin{array}{l}\text { Strengthen the lower back, hips and lower limbs prior to } \\
\text { competitive phases. Trunk stabilization whilst simultaneously } \\
\text { expressing large amounts of force through the lower limbs. }\end{array}$ & $\begin{array}{l}\text { Physical preparation to increase functional } \\
\text { capacity. Trunk conditioning to increase } \\
\text { capacity to undertake manual labor. }\end{array}$ \\
\hline $\begin{array}{l}\text { Core/lower back } \\
\text { Strength }\end{array}$ & Significant activation of the abdominals, oblique's and erector spina & muscles if deadlift loads are $>80 \%$ IRM. \\
\hline
\end{tabular}

\section{Muscle mechanics}

\section{Chest and triceps muscles activity}

Traditionally, the bench press is used to strengthen or develop the pectoralis major and the triceps, interestingly, the degree in which these muscles are activated depends on which variation is being performed. Peak activity of the pectoralis major a prime mover in the bench press is thought to peak in the later portion of the lowering phase and again early in the lifting period. For example, various studies have examined the effect different angled bench presses have on upper (clavicular portion) and lower (sternal portion) Pectoralis major activation. For the lower pectoralis major, research suggests both decline and flat bench; bench presses is optimal. For the upper pectoralis major, some research suggests bench angles between 28 to 56 degrees is optimal. However, other research has found no difference in the activation level of the clavicular portion of the pectoralis major when the bench press is performed on a flat and incline bench. Research has also found wider bench press grips stimulate more of the lower (sternal) pec attachments. ${ }^{57}$ For the clavicular portion pectoralis major, it appears narrower grips may be more effective. Research indicates anterior deltoid activity is most significant during incline (30 to 45 degrees) bench presses. Finally, for triceps, research suggests narrow grip bench presses are more efficient than medium and wider grip bench presses. Also, other researchers have observed, barbell bench presses may be superior to dumbbell bench presses for the triceps. Taken together, these results imply decline bench presses work the lower pectoralis major (sternal portion) and incline bench presses 
to target the upper pectoralis major (clavicular portion). Whereas, narrower grip barbell bench presses, may be more efficient for triceps muscle activation.

\section{Leg back and core muscle activity}

Many strength and conditioning coaches believe contraction of the latissimus dorsi during the lifting phase of the bench press is essential to performance. However, the involvement of the latissimus dorsi in the bench press has not been thoroughly investigated in trained lifters. In a previous study, the researchers reported the latisimus dorsi remained mostly inactive in inexperienced lifters. Powerlifters are known to hyperextend the spine during a bench press, which is thought to increase latissimus dorsi activity by declining the torso angle and altering muscle recruitment patterns around the shoulder. Interstingly, reported increased latissimus dorsi activity during a wider grip decline bench press. Hyperextending the spine during a flat bench press position the chest in slightly greater declined position, and thus, maybe one rationale explanation for this practice in competitive powerlifting events.

The bench press-benefits and application to sport and

Table 3 Outlined below are some examples of both sporting and ADLs tasks that may benefit

\section{activities of daily living}

Strength in the bench press may be necessary for successful performance in some different sports. Strength in the bench press may also be necessary for health and the ability to carry out activities of daily living. For instance, bench press strength differentiates professional from amateur rugby league players. Bench press strength is also strongly and significantly correlated to punching power in combat athletes. Finally, bench press strength is also associated with throwing performance in track and field events. Not only is upper body strength necessary for sporting performance, but also for improving the ability of older adults to carry out daily tasks such as carrying a box of groceries. From a health perspective, it has been found that performing upper body strengthening exercises including the bench press helps maintain muscle mass and strength with age, preventing some associated health conditions relating to low muscle mass. In the final analysis, the results of these studies strongly suggest bench press strength is essential for performance in sport, health and daily life. Upper body conditioning to increase capacity to undertake manual labor. Core/lower back Strength. Significant activation of the core muscles has been observed during the bench press, making the bench press a suitable assistance exercise to develop core strength (Table 3).

\begin{tabular}{|c|c|c|}
\hline $\begin{array}{l}\text { Physiological } \\
\text { Response/Benefit }\end{array}$ & Sporting Benefit & ADL/General Benefit \\
\hline $\begin{array}{l}\text { Hypertrophy of the arms } \\
\text { and chest. }\end{array}$ & $\begin{array}{l}\text { Increases effective mass and functional hypertrophy in the } \\
\text { upper body for contact sports. }\end{array}$ & Prevention of sarcopenia in older adults. \\
\hline $\begin{array}{l}\text { May increase or prevent } \\
\text { a decline in bone } \\
\text { mineral density. }\end{array}$ & $\begin{array}{l}\text { Important for Rugby League, Rugby Union and other contact } \\
\text { sports buffer collisions and contact. Assist speed/power athletes } \\
\text { with performance without the additional total body mass. }\end{array}$ & $\begin{array}{l}\text { Prevention/treatment for low bone mineral } \\
\text { density. }\end{array}$ \\
\hline $\begin{array}{l}\text { Whole body strength, } \\
\text { power and sports } \\
\text { performance }\end{array}$ & Rugby fends, tackling, punching power, shot put performance. & Carrying groceries, pushing trolleys. \\
\hline Rehabilitation & \multicolumn{2}{|c|}{$\begin{array}{l}\text { General rehabilitation and strengthening of the upper limbs following shoulder injuries and/or to condition the } \\
\text { upper limbs for a return to work or to upgrade functional capacity. }\end{array}$} \\
\hline Prehabilitation & $\begin{array}{l}\text { Strengthen the upper limbs (chest/arms) prior to competition. } \\
\text { Trunk muscle activation and stabilization whilst simultaneously } \\
\text { expressing large amounts of force through the upper limbs. }\end{array}$ & $\begin{array}{l}\text { Physical preparation to increase functional } \\
\text { capacity. Upper body conditioning to } \\
\text { increase capacity to undertake manual labor. }\end{array}$ \\
\hline $\begin{array}{l}\text { Core/lower back } \\
\text { Strength }\end{array}$ & \multicolumn{2}{|c|}{$\begin{array}{l}\text { Significant activation of the core muscles has been observed during the bench press, making the bench press an } \\
\text { suitable assistance exercise to develop core strength }\end{array}$} \\
\hline
\end{tabular}

\section{Acknowledgement}

None.

\section{Conflict of interest}

The authors declared that no conflict of interest exists.

\section{References}

1. Baker D. Comparison of upper-body strength and power between professional and college-aged rugby league players. J Strength Cond Res. 2000;15(1):30-35.

2. Wallace DA, Salem GJ, Salinas R, et al. Patello femoral joint kinetics while squatting with and without an external load. J Orthop Sports Phys Ther 2002;32(4):141-148.

3. Chandler TJ, Stone MH. The squat exercise in athletic conditioning:
A position statement and review of the literature. Chiropractic Sports Medicine. 1992;(6):105-105.

4. Kotani Y, Hori N. The reasons why athletes squat deep in japan volleyball women's national team. Journal of Australian Strength and Conditioning. $2017 ; 25(3)$.

5. Bloomquist K, Langberg H, Karlsen S, et al. Effect of range of motion in heavy load squatting on muscle and tendon adaptations. Eur J Appl Physiol. 2013;113(8):2133-2142.

6. Wisløff U, Castagna C, Helgerud J, et al. Strong correlation of maximal squat strength with sprint performance and vertical jump height in elite soccer players. British journal of sports medicine. 2004;38(3):285-288.

7. Rhea MR, Kenn JG, Peterson MD, et al. Joint-Angle Specific Strength Adaptations Influence Improvements in Power in Highly Trained Athletes. Human movement. 2016;17(1):43-49.

8. Caterisano A, Moss RE, Pellinger TK, et al. The effect of back squat depth 
on the EMG activity of 4 superficial hip and thigh muscles. $J$ Strength Cond Res. 2002;16(3):428-432.

9. Swinton PA, Stewart A, Agouris I, et al. A biomechanical analysis of straight and hexagonal barbell deadlifts using submaximal loads. $J$ Strength Cond Res. 2011;25(7):1-9.

10. Escamilla RF. Knee biomechanics of the dynamic squat exercise. Med Sci Sports Exerc. 2001;33(1):127-141.

11. Huck CJ. Effects of supervised resistance training on fitness and functional strength in patients succeeding bariatric surgery. J Strength Cond Res. 2015;29(3):589-595.

12. Amis A, Firer P, Mountney J, et al. Anatomy and biomechanics of the medial patellofemoral ligament. Knee. 2003;10(3):215-220.

13. Lefebvre R, Leroux A, Poumarat G, et al. Vastus medialis: anatomical and functional considerations and implications based upon human and cadaveric studies. J Manipulative Physiol Ther. 2006;29(2):139-144.

14. Karst GM, Willett GM. Onset timing of electromyographic activity in the vastus medialis oblique and vastus lateralis muscles in subjects with and without patellofemoral pain syndrome. Phys Ther. 1995;75(9):813-823.

15. Wallace DA, Salem GJ, Salinas R, et al. Patellofemoral joint kinetics while squatting with and without an external load. J Orthop Sports Phys Ther. 2002;32(4):141-148.

16. Anderson R, Courtney C, Carmeli E. EMG analysis of the vastus medialis vastus lateralis muscles utilizing the unloaded narrow-and wide-stance squats. Journal of Sport Rehabilitation. 1998;7(4):236-247.

17. Piper TJ, Waller MA. Variations of the Deadlift. Strength \& Conditioning Journal. 2001;23(3):1-66.

18. Wilk KE, Escamilla RF, Fleisig GS, et al. A comparison of tibiofemora joint forces and electromyographic activit during open and closed kinetic chain exercises. Am J Sports Med. 1996;24(4):518-527.

19. Blanpied PR. Changes in muscle activation during wall slides and squatmachine exercise. Journal of Sport Rehabilitation. 1999;8(2):123-134.

20. Rippetoe M. Starting Strength. The Aasgaard Company; 2017.

21. Almstedt HC, Canepa JA, Ramirez DA, et al. Changes in bone mineral density in response to 24 weeks of resistance training in college-age men and women. J Strength Cond Res. 2011;25(4):1098-1103.

22. Ariel BG. Biomechanical analysis of the knee joint during deep knee bends with heavy load. In: Nelson RC, Morehouse CA, editors. Biomechanics IV. International Series on Sport Sciences. Palgrave, London; 1974:44-52.

23. Barnett C, Kippers V, Turner P. On the EMG Activity of Five Shoulder Muscles. Journal of Strength and Conditioning Research. 1995;9(4):222-227.

24. Bejjani F, Gross C, Pugh J. Model for static lifting: relationship of loads on the spine and the knee. Journal of biomechanics. 1984;17(4):281-286.

25. Bezerra ES, Simão R, Fleck SJ, et al. Electromyographic activity of lower body muscles during the deadlift and still-legged deadlift. Journal of Exercise Physiology Online. 2013;16(3):30-39.

26. Braidot A, Brusa M, Lestussi F, et al. Biomechanics of front and back squat exercises. Paper presented at the Journal of Physics: Conference Series; 2007.

27. Cholewicki J, Mc Gill S, Norman R. Lumbar spine loads during the lifting of extremely heavy weights. Med Sci Sports Exerc. 1991;23(10):1179-1186.

28. Contreras B, Vigotsky AD, Schoenfeld BJ, et al. A comparison of gluteus maximus, biceps femoris, and vastus lateralis electromyography amplitude in the parallel, full, and front squat variations in resistance-trained females. J Appl Biomech. 2016;32(1):16-22.
29. Del Vecchio L, Stanton R, Reaburn P. Maximal upper body strength and power are strongly correlated in amateur male kickboxers. Paper presented at the the International Conference on Applied Strength and Conditioning, Melbourne, Victoria; 2013.

30. Dobek JC, White KN, Gunter KB. The effect of a novel ADL-based training program on performance of activities of daily living and physical fitness. J Aging Phys Act. 2007;15(1):13-25.

31. Duffey MJ. A biomechanical analysis of the bench press: The Pennsylvania State University; 2008.

32. Ebben WP, Feldmann CR, Dayne A, et al. Using squat testing to predict training loads for the deadlift, lunge, step-up, and leg extension exercises. J Strength Cond Res. 2008;22(6):1947-1949.

33. Escamilla RF, Francisco AC, Kayes AV, et al. An electromyographic analysis of sumo and conventional style deadlifts. Med Sci Sports Exerc. 2002;34(4):682-688

34. Farley K. Analysis of the Conventional Deadlift. Strength \& Conditioning Journal. 1995;17(6):55-57.

35. Freivalds A, Chaffin DB, Garg A, et al. A dynamic biomechanical evaluation of lifting maximum acceptable loads. J Biomech. 1984;17(4):251-262.

36. Gomo O, Van Den Tillaar R. The effects of grip width on sticking region in bench press. J Sports Sci. 2016;34(3):232-238.

37. Green CM. The affect of grip width on bench press performance and risk of injury. Strength and Conditioning Journal. 2007:29(5):1-10.

38. Hales ME, Johnson BF, Johnson JT. Kinematic analysis of the powerlifting style squat and the conventional deadlift during competition: is there a crossover effect between lifts? J Strength Cond Res. 2009;23(9):2574-2580.

39. Hartmann H, Wirth K, Klusemann M, et al. Influence of squatting depth on jumping performance. The J Strength Cond Res. 2012;26(12):3243-3261.

40. Horn T. A biomechanical comparison of sumo and conventional deadlifting techniques. Paper presented at the International Journal of Sports Medicine; 1988.

41. Hunter GR, Treuth MS, Weinsier RL, et al. The effects of strength conditioning on older women's ability to perform daily tasks. $J$ Am Geriatr Soc. 1995;43(7):756-760.

42. Law TD, Clark LA, Clark BC. Resistance exercise to prevent and manage sarcopenia and dynapenia. Annu Rev Gerontol Geriatr. 2016;36(1):205.

43. Madsen N, Mclaughlin T. Kinematic factors influencing performance and injury risk in the bench press exercise. Med Sci Sports Exerc. 1984;16(4):376-381.

44. Mc Cartney N, Hicks AL, Martin J, et al. Long-term resistance training in the elderly: effects on dynamic strength, exercise capacity, muscle, and bone. The Journals of Gerontology Series A: Biological Sciences and Medical Sciences. 1995;50(2):97-104.

45. Mc Guigan MR, Wilson BD. Biomechanical Analysis of the Deadlift. The Journal of Strength \& Conditioning Research. 1996;10(4):250-255.

46. Noe DA, Mostardi RA, Jackson ME, et al. Myoelectric activity and sequencing of selected trunk muscles during isokinetic lifting. Spine. 1992;17(2):225-229.

47. Paoli A, Marcolin G, Petrone N. The effect of stance width on the electromyographical activity of eight superficial thigh muscles during back squat with different bar loads. J Strength Cond Res. 2009;23(1):246-250.

48. Patrick T, Bellar D, Judge L, et al. Correlation of Height and Preseason Bench Press 1Rm to Shot Put and Weight Throw Performance During the Competitive Season. The Journal of Strength \& Conditioning Research. 2011;(25):97-98. 
49. Saeterbakken AH, Vanden Tillaar R, Fimland MS. A comparison of muscle activity and 1-RM strength of three chest-press exercises with differen stability requirements. J Sports Sci. 2011;29(5):533-538.

50. Schipplein O, Trafimow J, Andersson G, et al. Relationship between moments at the L5/S1 level, hip and knee joint when lifting. J Biomech. 1990;23(9):907-912.

51. Schultz AB, Andersson GB. Analysis of loads on the lumbar spine. Spine. 1981;6(1):76-82.

52. Suprak DN, Dawes J, Stephenson MD. The effect of position on the percentage of body mass supported during traditional and modified pushup variants. J Strength Cond Res. 2011;25(2):497-503.

53. Thompson BJ, Stock MS, Shields JE, et al. Barbell deadlift training increases the rate of torque development and vertical jump performance in novices. J Strength Cond Res. 2015;29(1):1-10.
54. Vanden Tillaar R, Ettema, G. A comparison of successful and unsuccessful attempts in maximal bench pressing. Med Sci Sports Exerc. 2009;41(11):2056-2063.

55. Wagner LL, Evans SA, Weir JP, et al. The effect of grip width on bench press performance. International Journal of Sport Biomechanics. 1992;8(1):1-10.

56. Young WB. Transfer of strength and power training to sports performance. Int J Sports Physiol Perform. 2006;1(2):74-83.

57. Zweifel M. Importance of Horizontally Loaded Movements to Sports Performance. Strength \& Conditioning Journal. 2017;39(1):21-26. 